\title{
Блазар АО 0235+164 - тесная система из сверхмассивных черных дыр
}

\author{
А.Е. Вольвач ${ }^{1}$, М.Г. Ларионов ${ }^{2}$, Л.Н. Вольвач${ }^{1}$ \\ 1 ФГБУН “Крымская астрофизическая обсерватория РАН”, Научный, Крым, 298409 \\ volvach@craocrimea.ru \\ 2 Астрокосмический центр Физического института им. П.Н. Лебедева РАН, Москва, Россия
}

Поступила в редакцию 16 марта 2018 г.

Аннотация. Выполнен анализ данных многочастотных наблюдений блазара АО 0235+164 в радиодиапазоне. Обнаружены свидетельства о двойственности системы, состоящей из двух сверхмассивных черных дыр, находящихся на стадии эволюции, близкой к слиянию. С помощью гармонического анализа установлены возможные значения орбитального и прецессионного периодов в системе, аналогично тем, которые получены для других ярких представителей АЯГ. Сдвиги по времени вспышечных явлений, возникающих в разных частотных диапазонах, соответствуют гипотезе перемещающегося по джету плазменного облака, просветляющегося последовательно на все более низких частотах, что указывает скорее на внутренний характер переменности плотности потока в АО 0235+164. Найдены физические и динамические характеристики АЯГ АО 0235+164, свидетельствующие о том, что эта тесная система состоит из СЧД близких масс порядка $10^{10} \mathrm{M}_{\odot}$.

BLAZAR AO 0235+164 - A CLOSE SYSTEM OF SUPERMASSIVE BLACK HOLES, by A.E. Volvach, M.G. Larionov, L.N. Volvach. The data of multi-frequency observations of the blazar $\mathrm{AO} 0235+164$ in radio waves ranges are analyzed. Evidence of the duality of a system consisting of two supermassive black holes, which are at the stage of evolution close to fusion, is found. With the help of harmonic analysis, possible values of the orbital and precession periods in the system are established, similar to those obtained for other bright representatives of AGN. The time shifts of flare phenomena occurring in different frequency ranges correspond to the hypothesis of a plasma cloud moving along the jet, which is bleached sequentially at lower and lower frequencies, what indicates rather the internal nature of variability of the flux density in $\mathrm{AO} 0235+164$. The physical and dynamic characteristics of AGN AO $0235+164$ suggest that this close system consists of supermassive black holes with similar masses of the order of $10^{10} \mathrm{M}_{\odot}$.

Ключевые слова: активные ядра галактик, радиоизлучение, переменность плотности потока

\section{1 Введение}

Активное ядро галактики АО 0235+164, обладающее характеристиками блазара, относится к ярчайшим АЯГ, которые активно исследуются в широком диапазоне длин волн на многих обсерваториях мира. В объекте зафиксированы значительные изменения плотности потока излучения на различных 
временных масштабах - от долей часов до десятков лет. Как и в других выдающихся АЯГ, в источнике АО 0235+164 наблюдаются сверхкомпактные структурные образования (Джорстад и др., 2001; Спинрад, Смит, 1975). Такие детали имеют высокую яркостную температуру и обладают сверхсветовыми движениями (Рике и др., 1976; Чен и др., 1999; Фрей и др., 2000). В них отмечена высокая поляризация радио- и оптического излучения. Именно релятивистскими доплеровскими эффектами объясняются предельно высокие яркостные температуры, получаемые по данным интерферометрических наблюдений и измерений на одиночных антеннах (Раитери и др., 2005; Блэндфорд, Кёнигл, 1979).

Долговременный мониторинг АО 0235+164 на одиночных антеннах начался в 1975 г. в Мичиганской радиоастрономической обсерватории (США). Многочисленные наблюдения источника в рентгеновском диапазоне выполнялись с 1990 г. (Воррал, Вилкес, 1990), а в гамма-диапазоне регулярные данные поставляет космический телескоп Fermi LAT (Large Area Telescope) ${ }^{1}$. Из оптических и радиокривых блеска обнаружен квазипериод 5.7 лет (Клементс и др., 1995). С тех пор этот объект стал хорошим кандидатом на двойную систему из СЧД (Ромеро и др., 2000). В дальнейшем нашими исследованиями было подтверждено такое утверждение и, более того, мы предположили существование тесных двойных систем из СЧД, которые интенсивно излучают только в том случае, когда компаньон центральной СЧД проходит сквозь аккреционный диск (АД) центрального тела и возмущает его (Вольвач и др., 2007а). Из-за прецессии центрального тела орбитальный период может исчезать по причине изменения угла между выбросами и направлением на наблюдателя. В рассматриваемой модели в системе должны присутствовать прецессионный и орбитальный периоды.

\section{2 Наблюдения}

22-метровый радиотелескоп в Симеизе использовался для проведения наблюдательной программы на частотах 22.2 и 36.8 ГГц. На входе радиотелескопа стояли модуляционные радиометры, работающие в режиме диаграммной модуляции, при котором с частотой 1 кГц производилось переключение приемных рупоров и осуществлялся разностный прием излучения с близких по положению точек небесной сферы. Экспозиция на источнике изменялась в пределах (5-100) сек в зависимости от спектральной плотности потока излучения исследуемого объекта. Затухание в атмосфере Земли измерялось с интервалами времени два-три часа с помощью метода “разреза атмосферы”. Процедура заключалась в определении значений антенной температуры на фиксированных углах места радиотелескопа, между которыми были известны отношения уровней поглощения. Последние выражались в единицах высоты однородной атмосферы. Температурная “привязка” наблюдательных данных производилась при помощи калиброванных шумовых сигналов, которые подавались на входы приемников. Дополнительно они использовались для контроля усиления приемных трактов.

Поправки наведения радиотелескопа на источник определялись методом сканирования антенны по прямому восхождению $\alpha$ и склонению $\delta$. Обработка поступающей информации осуществлялась в режиме on-line с использованием пакета программ, разработанного в обсерватории.

На рис. 1 приведены данные длительного многочастотного мониторинга АО 0235+164 на частотах 22, 37 ГГц (с 1981 г.). Видна ярко выраженная вспышечная активность блазара.

\section{3 Анализ данных}

Гармонический анализ вариаций потоков АО 0235+164 на различных длинах волн был выполнен методом Шустера и изложен в работе (Вольвач и др., 2013). Максимальный период длительностью 8.5 лет ярко выражен на частотах 22.2 и 36.8 ГГц. Этот период может быть прецессионным, как и в случае других ярких АЯГ (Вольвач и др., 2012). Разброс в значениях периода не выходит за пределы статистических погрешностей. Кроме того, могут различаться физические условия возникновения излучения в объекте на разных частотах. Короткий период (около двух лет) можно связать с орбитальным периодом движения компаньона вокруг общего центра тяжести в системе, состоящей из

${ }^{1}$ http://fermi.gsfc.nasa.gov/ssc/data/analysis/scitools/extract_latdata.html 


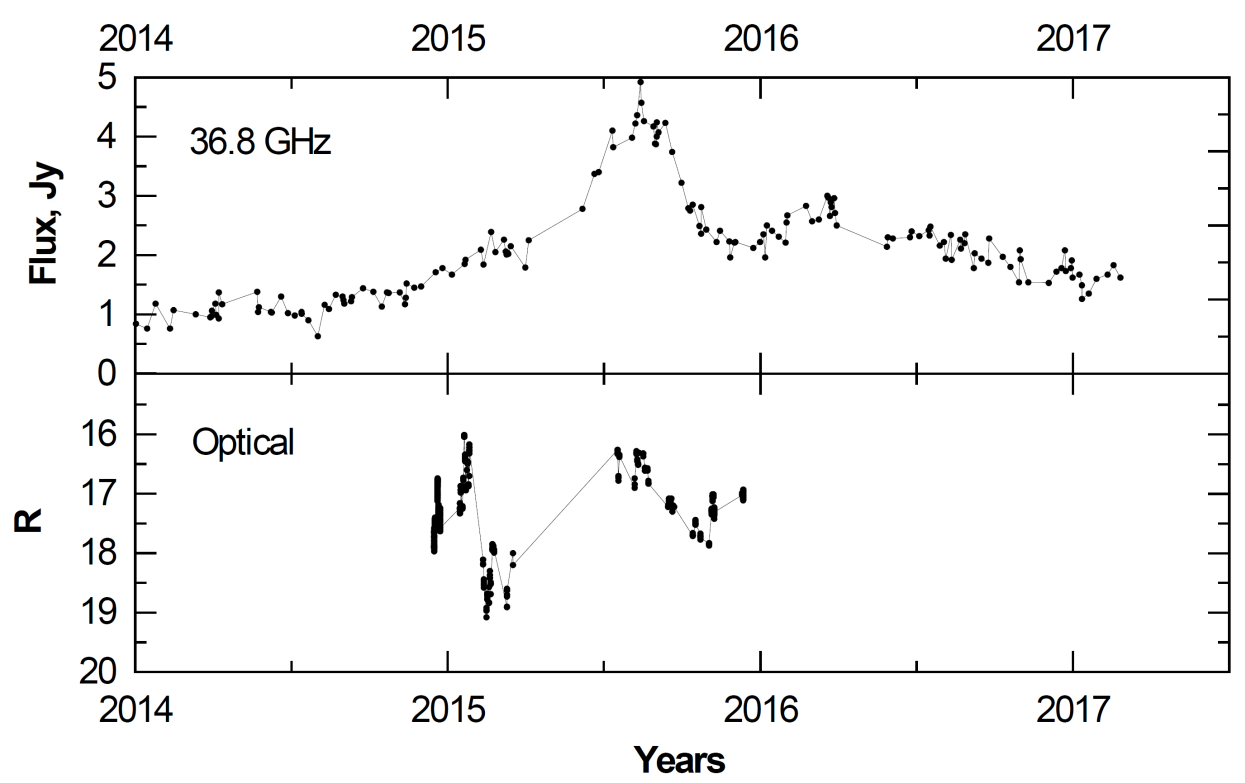

Рис. 1. Вспышечные явления в $\mathrm{AO} 0235+164$ в оптическом и радиодиапазонах

двух СЧД. Существует набор промежуточных комбинационных периодов от 3 до 5.5 лет, которые могут объясняться вариациями в движениях системы, состоящей более чем из двух тел. Такая система включает в себя две СЧД, АД, погруженные в неоднородную плотную аккрецирующую среду.

\section{4 Сдвиги по времени между вспышечными явлениями на миллиметровых волнах}

Вспышечные явления в АЯГ являются следствием активности в их внутренних областях. Как уже было сказано, такая активность в АЯГ может быть связана с пересечением компаньона центральной СЧД ее АД. Передача активности в “джеты” может происходить посредством ударных волн. Методами интерферометрии со сверхдлинными базами (РСДБ) и наблюдениями на одиночных антеннах мы такую активность наблюдаем в АЯГ (Вольвач и др., 2009). Наблюдаемые сверхсветовые движения компонент в выбросах происходят с задержками по времени между различными частотными диапазонами. Это естественно принять, если допустить, что в процессе распространения облако плазмы расширяется и становится наблюдаемым на более низких частотах. Характер задержек вспышек дает информацию о характеристиках среды “джетов" (их магнитном поле) и о динамике системы из двойных СЧД. Зависимость задержек вспышек на разных частотах в работе (Вольвач и др., 2015) была аппроксимирована обратнологарифмической зависимостью:

$$
\Delta T=\frac{k}{\log \nu},
$$

где $\Delta T$ - задержка, выраженная в годах от начала вспышки в высокочастотном диапазоне, $\nu$ - частота наблюдений в ГГц, $k$ - константа, характеризующая крутизну наклона зависимости. Задержки явлений между гамма-диапазоном и оптикой находились в пределах ошибок измерений, составлявших несколько дней.

Для вспышек, проанализированных работе (Вольвач и др., 2015), было получено значение $k=$ 0.25 , что соответствовало задержке между оптикой и радиодиапазоном около двух месяцев. 
Временные сдвиги между вспышечными явлениями на частотах 22.2 и 36.8 ГГц определялись кросскорреляционным методом, детали которого изложены в работе (Вольвач и др., 2007б).

Для вспышечного явления 2015-2016 гг. с использованием выражения (1) нами было получено значение (экстраполяция зависимости) для коэффициента $k=0.12$, что соответствует задержке между оптическим и радиодиапазонами (37 ГГц) $28 \pm 2$ дня.

Из анализа наблюдательных данных нами в (Вольвач и др., 2014) высказывалось предположение о том, что задержки вспышечных явлений на разных частотах в разных АЯГ находятся в квадратичной зависимости от углов между направлением выбросов и лучом зрения наблюдателя. Этот угол в АО $0235+164$ находится в пределах $\left(2-3^{\circ}\right)$. По данным работы (Кутькин и др., 2018) он равен $\theta=2.9^{\circ}$, что соответствует $\gamma \approx 20$. Эту величину можно принять за консервативное значение лоренц-фактора в АО $0235+164$, и она была использована нами при определении периодов в системе отсчета источника.

Для случая вспышки 2015 г. соответствующие задержки оказались вдвое меньше, чем для вспышки 2008 года. Так, задержка между оптическим и радиодиапазоном (37 ГГц) составила 28 дней. Это означает, что угол между выбросом из АО $0235+164$ и направлением на наблюдателя $-\theta-$ стал в 1.5 раза меньше, то есть $\theta=\left(1.4-1.7^{\circ}\right)$.

\section{5 Заключение}

Проанализированы данные длительных многочастотных наблюдений блазара АО 0235+164, выполненных на РТ-22 КрАО, обсерватории Метсахови и в гамма-диапазоне (космическая обсерватория Ферми).

Подтверждены аргументы в пользу двойственности системы АО 0235+164, состоящей из двух СЧД. Гармоническим анализом установлены возможные значения орбитального и прецессионного периодов, аналогичные выявленным у других ярких представителей АЯГ. Дополнительные комбинационные частоты могут быть следствием присутствия возможного нутационного периода и удвоенных значений обнаруженных периодов.

Изменения, зафиксированные в АО $0235+164$, являются следствием уменьшения угла $\theta$ между выбросами из блазара и направлением на наблюдателя в $\sqrt{2}$ раз. Меньшее значение угла $\theta$ зафиксировано только в одном экстремальном АЯГ - S5 0716+714.

\section{Литература}

Блэндфорд, Кёнигл (Blandford R.D., Königl A.) // Astrophys. J. 1979. V. 232. P. 34.

Вольвач А.Е., Вольвач Л.Н., Ларионов М.Г. и др. // Астрон. журн. 2007а. Т. 84. № 6. С. 503.

Вольвач А.Е., Юровский Ю.Ю., Вольвач Л.Н., Стрепка И.Д. // 17-я Международная конференция

“СВЧ-техника и телекоммуникационные технологии". 2007б. Севастополь. Крым. С. 824.

Вольвач А.Е., Пушкарев А.Б., Вольвач Л.Н., и др. // Космическая наука и технология. 2009. Т. 15. № 4. C. 33 .

Вольвач и др. (Volvach A.E., Volvach L.N., Bychkova V.S., et al.) // Astron. Rep. 2012. V. 56. P. 275.

Вольвач А.Е., Кутькин А.М., Вольвач Л.Н. и др. // Астрон. журн. 2013. Т. 90. № 1. С. 40.

Вольвач А.Е., Кутькин А.М., Ларионов М.Г. и др.// Астрон. журн. 2014. Т. 91. № 2. С. 122.

Вольвач А.Е., Ларионов М.Г., Вольвач Л.Н. и др. // Астрон. журн. 2015. Т. 92. № 2. С. 168.

Воррал, Вилкес (Worrall D.M., Wilkes B.J.) // Astrophys. J. 1990. V. 360. P. 396.

Джорстад и др. (Jorstad S.G., Marscher A.P., Mattox J.R., et al.) // Astrophys. J. 2001. V. 134. P. 181.

Клементс и др. (Clements S.D., Smith A.G., Aller H.D., and Aller M.F.) // Astron. J. 1995. V. 110. P. 529.

Кутькин и др. (Kutkin A.M., Pashchenko I.N., Lisakov M.M., et al.) // Mon. Not. Roy. Astron. Soc. 2018. V. 475. Issue 4. P. 4994.

Раитери и др. (Raiteri C.M., Villata M., Ibrahimov M.A., et al.) // Astron. Astrophys. 2005. V. 438. P. 39.

Рике и др. (Rieke G.H., Grasdalen G.L., Kinman T.D., et al.) // Nature. 1976. V. 260. P. 754. 
Ромеро и др. (Romero G.E., Sellone S.A., Combi J.A.) // Astron. Astrophys. 2000. V. 360. P. 47. Спинрад, Смит (Spinrad H. and Smith H.) // Astrophys. J. 1975. V. 201. P. 275.

Фрей и др. (Frey S., Gurvits L.I., Altschuller R.D., et al.) // Publ. Astron. Soc. Pacific. 2000. V. 52. P. 975.

Фуджисава и др. (Fujisawa K., Kobajashi H., Wajima K., et al.) // Publ. Astron. Soc. Japan. 1999. V. 51. P. 537.

Чен и др. (Chen Y.J., Zhang F.Y., and Sjouwermen L.O.) // Astrophys. Space Sci. 1999. V. 266. P. 495. 